\title{
Deconvolution of positron annihilation coincidence Doppler broadening spectra using an iterative projected Newton method with non-negativity constraints
}

\author{
K. F. Ho, C. D. Beling, S. Fung, a) and Vincent K. W. Cheng \\ Department of Physics, The University of Hong Kong, Pokfulam Road, Hong Kong, \\ People's Republic of China \\ Michael K. Ng \\ Department of Mathematics, The University of Hong Kong, Pokfulam Road, Hong Kong, \\ People's Republic of China
}

\author{
A. M. Yip \\ Department of Mathematics, University of California, Los Angeles, 405 Hilgard Avenue, Los Angeles, \\ California 90095
}

(Received 21 March 2003; accepted 18 August 2003)

\begin{abstract}
A generalized least-square method with Tikonov-Miller regularization and non-negativity constraints has been developed for deconvoluting two-dimensional coincidence Doppler broadening spectroscopy (CDBS) spectra. A projected Newton algorithm is employed to solve the generalized least-square problem. The algorithm has been tested on Monte Carlo generated spectra to find the best regularization parameters for different simulated experimental conditions. Good retrieval of the underlying positron-electron momentum distributions in the low momentum region is demonstrated. The algorithm has been successfully used to deconvolute experimental CDBS data from aluminum. (C) 2003 American Institute of Physics. [DOI: 10.1063/1.1619547]
\end{abstract}

\section{INTRODUCTION}

Angular correlation of annihilation radiation (ACAR) and Doppler broadening spectroscopy (DBS) provide information on the electron momentum distribution (EMD) in the material under investigation through measurements of the momentum density of electron-positron annihilating pairs (MDAP) ${ }^{1}$ In regular solids the MDAP can be used to check theoretical band structure calculations, and provides details on Fermi surfaces in more complex alloys. ${ }^{2}$ The higher resolving power of ACAR is normally required for these studies. On the other hand, in defected solids where the MDAP is normally more representative of the positron trapping sites than the bulk solid, the DBS technique can be quickly and usefully employed to distinguish different defects by plotting the $S$ (valence) parameter against the $W$ (core) parameter (the $S-W$ plot). ${ }^{3}$ The DBS technique, while capable of a fast data accumulation rate, suffers from the poor intrinsic resolution of $\mathrm{HP}(\mathrm{Ge})$ gamma ray spectroscopy systems (typically, $\sim 5$ mrad ACAR equivalent, in comparison to $\sim 1$ mrad for typical ACAR systems). DBS on its own is thus normally considered insufficient for accurate MDAP measurement in regular solids, and with regard to defect studies DBS is often perceived to have reached its limits of usefulness in the $S-W$ plot.

An extension of the conventional DBS, coincidence Doppler broadening spectroscopy (CDBS), has recently been opening up new horizons. ${ }^{4}$ In CDBS, two simultaneous measurements are made of the Doppler shift on a pair of annihi-

a)Electronic mail: sfung@ @kucc.hku.hk lation photons; a procedure having the advantage of providing very low background levels, which permits core annihilations to be accurately observed, and thus providing information on the chemical environment of the positron. ${ }^{5}$ Moreover, with relevance in MDAP measurement, CDBS also has the intrinsic property of improving the instrumental resolution by factors of up to $\sqrt{2}(\sqrt{2}$ applying only when the detectors have equal resolution), ${ }^{6}$ together with the possibility of high quality deconvolution being made possible by the fact that an almost perfect resolution function of the system exists; namely, in the form of the energy spectrum of the 514 $\mathrm{keV}$ gamma ray line of ${ }^{85} \mathrm{Sr}$, where photons from the source are observed in pseudocoincidence. ${ }^{7}$ Britton et al. $^{7}$ demonstrated both the importance of the $\sqrt{2}$ improvement and the effect of deconvolution by building a CDBS system with an effective resolution of $386 \mathrm{eV}$ in full width at half maximum (FWHM) unit ( $\sim 1.5 \mathrm{mrad}$ ACAR equivalent). The main motivation of the present work has been similar, namely, that of trying to investigate if ACAR quality spectra can be obtained using CDBS. CDBS remains a desirable method over ACAR because of its simplicity of operation and the added usefulness of the chemical environment sensitivity provided by its ability to observe high momentum core electron momenta.

The use of suitable deconvolution algorithms is an important issue in improving the quality of CDBS spectra in the low momentum range. A major factor in the success of any deconvolution venture is the quality of the input spectrum itself. Assuming the spectrum has been perfectly stabilized against electronic drift effects, there are still the uncertainties due to noise arising from the stochastic nature of the counting process. Thus, the more counts in the spectrum, the more 
true to the convoluted functional shape, i.e., the less noisy, it becomes. The number of channels into which the CDBS spectrum is discretized is also a factor effecting spectral quality. ${ }^{8}$ A well set up CDBS system could be expected to record good annihilation photon coincidences at a rate of $10^{3} \mathrm{cp} \mathrm{s}$, which over a period of a few days could give total number of counts $\left(N_{\text {cts }}\right) \sim 10^{9}$ events. ${ }^{8}$ Moreover, a good modern nuclear analog-to-digital converter (ADC) can digitize into number of channels $\left(N_{\text {chn }}\right) \sim 16000$, giving CDBS image data of $16000 \times 16000$ pixels. Improvements in deconvolution are not linear in $N_{\text {cts }}$ and $N_{\text {chn }}$ but tend to be logarithmic. ${ }^{8}$ The question which naturally arises as to what constitutes a "good enough" deconvolution under the presently available hardware and computational resources is one of the issues considered in the present work.

A variety of algorithms have been adopted for deconvolution studies on the one-dimensional (1D) annihilation line spectra of DBS in the past. Many different algorithms have been used such as the Stokes method, ${ }^{7,9-12}$ the maximum entropy method, ${ }^{13,14}$ iterative methods, ${ }^{15-18}$ optimized linear filtering, ${ }^{19}$ and the method of generalized least squares. ${ }^{20-24}$ It is the latter method which is presented in detailed form in this article. Although the methods listed above have all given promising results, none of them, excepting Ref. 23, incorporates non-negativity constraints on the deconvoluted spectra. A major objective of the present work is to discuss the feasibility of implementing such constraints through the use of the projected Newton method. ${ }^{25}$ Another objective is to explore the more general use of deconvolution in the twodimensional (2D) image data produced in CDBS.

The outline of the article is as follows: First, with reference to the intrinsically ill-conditioned image restoration problem, the use of Tikhonov regularization will be illustrated. A priori knowledge of non-negativity will be introduced into the generalized least-square formulation. A projected Newton-based algorithm will then be described to solve the least-square problem. The effectiveness of this algorithm is investigated as a function of the number of counts, matrix size (number of channels), order of derivative to be regularized, and the regularization parameter. The effective system resolution of a CDBS system which incorporates a deconvolution code is then considered. Finally, the projected Newton algorithm is applied to real experimental CDBS data for polycrystalline aluminum.

\section{THEORY}

\section{A. CDBS "image" data}

In the CDBS technique the energies $E_{1}$ and $E_{2}$ of both annihilation photons are measured by two $\mathrm{HP}(\mathrm{Ge})$ detectors in back-to-back orientation and $E_{2}$ is plotted against $E_{1}$ to form a $2 \mathrm{D}$ histogram. These energies may be written as

$$
\begin{aligned}
& E_{1}=m_{0} c^{2}-\frac{1}{2} E_{n}+\Delta E+\delta_{1}, \\
& E_{2}=m_{0} c^{2}-\frac{1}{2} E_{n}-\Delta E+\delta_{2},
\end{aligned}
$$

where $m_{0}$ is the electron rest mass, $E_{n}$ is the binding energy of electrons in the $n$th shell, $\Delta E$ is the Doppler shift, and $\delta_{1}$ and $\delta_{2}$ are the independent measurement errors for each de- tector. Annihilation events as given by Eq. (1) are thus seen as lying on the lines $E_{1}+E_{2}=2 m_{0} c^{2}-E_{n}$ with the lines broadened by $\sigma_{1}$ and $\sigma_{2}$ in both coordinates, the latter being the standard deviations of instrumental errors $\delta_{1}$ and $\delta_{2}$.

In comparison with conventional DBS method, the CDBS momentum signal is doubled since:

$$
E_{1}-E_{2}=p_{\|} c=2 \Delta E+\delta_{1}+\dot{\delta}_{2} .
$$

By taking $\sigma_{1}=\sigma_{2}$, one has, assuming $\delta_{1}$ and $\delta_{2}$ to be Gaussian in distribution, a standard deviation on $E_{1}-E_{2}$ of

$$
\sigma=\sqrt{\sigma_{1}^{2}+\sigma_{2}^{2}}=\sqrt{2} \sigma_{1} .
$$

From Eqs. (2) and (3), it is seen that the signal strength is doubled but the error on the signal has only increased by a factor of $\sqrt{2} \sim 40 \%{ }^{6}$ In practice, the condition $\sigma_{1}=\sigma_{2}$ is seldom met (the detectors being of different intrinsic resolution) so that the $\sqrt{2}$ improvement in momentum measurement is only approached, but never fully attained.

Figure 1(a) shows an experimental CDBS taken for polycrystalline aluminum. We can see that there are vertical and horizontal cross pieces at $E_{1}=m_{0} c^{2}$ and $E_{2}=m_{0} c^{2}$. These are produced on the high energy side by pulse pileup and on the low energy side by incomplete charge collection. In the case of the positron source being ${ }^{22} \mathrm{Na}$, events are present on the low and high energy sides due to coincident Compton events from the $1.27 \mathrm{MeV}$ gamma ray associated with this source. However, the cross pieces need not be of undue concern since in the first place their intensity is much less than that of the real annihilation events on the diagonal, and second, under the proviso that we have "subtracted off" any Compton background, the remaining counts can in some sense be considered as part of the instrumental resolution function, the features being connected with the main signal in the same way for both the CDBS spectrum and the instrumental "blurring" function. The presence of similar "cross pieces" indeed may be seen in the resolution spectrum shown in Fig. 1(b) obtained from a ${ }^{85} \mathrm{Sr}$ source taken under pseudocoincidence conditions.

\section{B. Deconvolution problem}

The convoluted 2D image $g_{i j}$ of a true 2D image function $f_{i j}(1 \leqslant i \leqslant m, 1 \leqslant j \leqslant m)$ with an instrumental function $h_{i j}(1 \leqslant i \leqslant m, 1 \leqslant j \leqslant m)$ with additive noise may be written ${ }^{26}$ as follows:

$$
g_{i j}=\sum_{i^{\prime}=1}^{m} \sum_{j^{\prime}=1}^{m} h_{i-i^{\prime} j-j^{\prime}} f_{i^{\prime} j^{\prime}}+n_{i j},
$$

where $n_{i j}$ is an unknown noise and $m$ is the size of the image ( $N_{\mathrm{chn}}$, in our case). The matrix form becomes

$$
\mathbf{g}=\mathbf{H f}+\mathbf{n} \text {. }
$$

It is well known that the matrix $\mathbf{H}$ is ill conditioned as a result of its averaging effect. Tikhonov ${ }^{27}$ postulated an equation:

$$
\underset{f}{\min }\|\mathbf{g}-\mathbf{H f}\|_{2}^{2}+\alpha\left\|\mathbf{f}^{(\mathbf{k})}\right\|_{2}^{2} .
$$



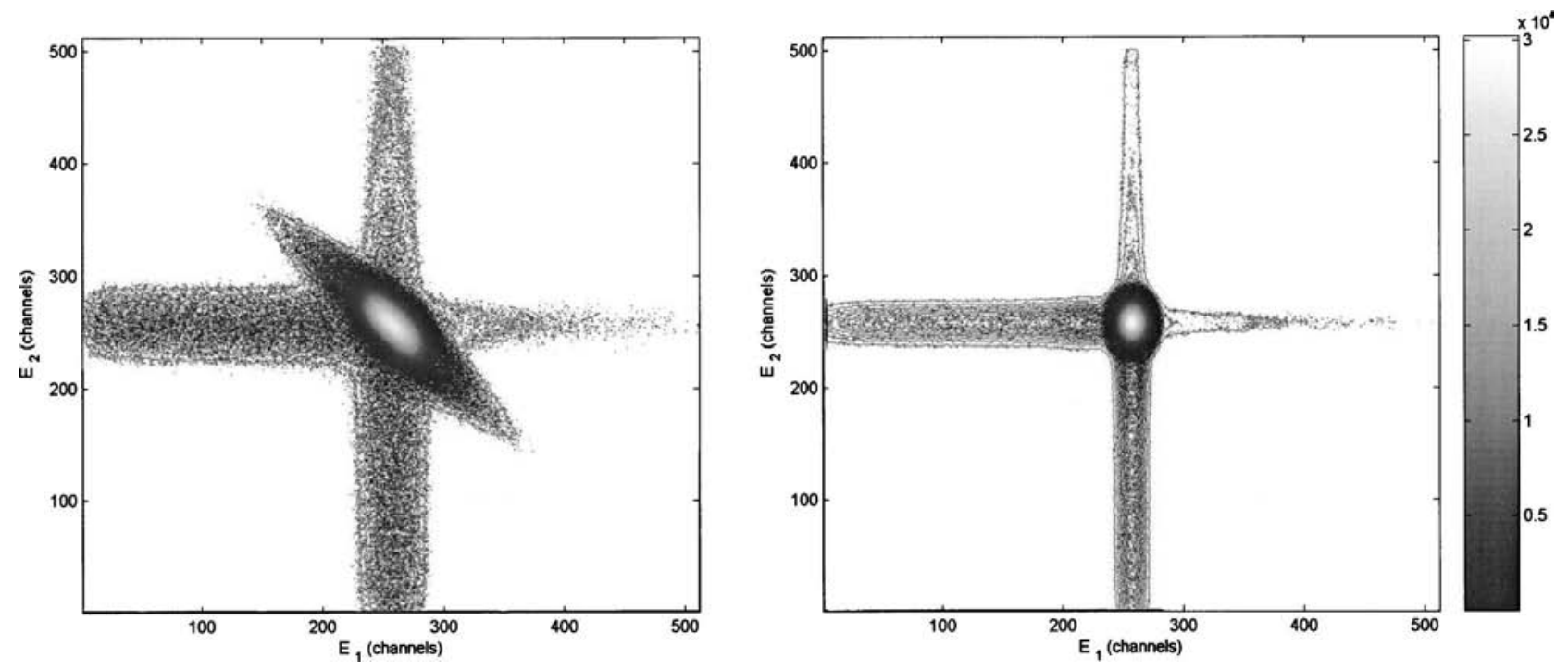

FIG. 1. (a) Experimental CDBS 2D histogram spectrum taken for polycrystalline $\mathrm{Al}$ metal $\left(N_{\mathrm{cts}}=2 \times 10^{7}\right)$. (b) Effective CDBS $2 \mathrm{D}$ histogram resolution function as obtained using a ${ }^{85} \mathrm{Sr}$ source $\left(E_{\gamma}=514 \mathrm{keV}\right)$ taken with random events (coincidence time window $\left.=2 \mu \mathrm{s}, N_{\mathrm{cts}}=5 \times 10^{7}\right)$. The energy calibration for both spectra is $75 \mathrm{eV} /$ channel. As can be seen, the resolution of the $E_{1}$ detector $(1.2 \mathrm{keV})$ is superior to that of the $E_{2}$ detector $(2.0 \mathrm{keV})$.

Here $\|\cdot\|_{2}$ denotes the Euclidean norm. In the minimization problem associated with Eq. (6), the quality of the solution is controlled by the choice of the regularization parameter $\alpha$ $>0$ in the second "Tikhonov regularization" term. Here, $\left\|\mathbf{f}^{\mathbf{( k )}}\right\|$ provides a measure of the total energy of $\mathbf{f}$ (when $k$ $=0$ ) or the $k$ th derivatives of $\mathbf{f}$ (when $k>0$ ), depending upon the particular choice of $k$. Large values of $\alpha$ yield less noisy solutions for $\mathbf{f}$ but with some loss of information on "sharp" features, while if $\alpha$ is set too low the solution for $\mathbf{f}$ may be too noisy. ${ }^{21}$ The formal solution of this equation is, for a given $\alpha$,

$$
\mathbf{f}_{\alpha}=\left(\mathbf{H}^{T} \mathbf{H}+\alpha \mathbf{L}^{(\mathbf{k})}\right)^{-1} \mathbf{H}^{T} \mathbf{g} .
$$

Here, $\mathbf{H}^{T}$ denotes the transpose of $\mathbf{H}$ and $\mathbf{L}^{(\mathbf{k})}$ is the regularization matrix corresponding to the $k$ th derivative of $\mathbf{f}$. If the instrumental function is spatially shift invariant, then the deconvoluted image $\mathbf{f}_{\alpha}$ can be obtained efficiently by using fast cosine or Fourier transforms. However, when the instrumental function is spatially shift variant, Eq. (7) can be solved iteratively to obtain $\mathbf{f}_{\alpha} \cdot{ }^{28}$

Both the calculated spectrum $\mathbf{f}$ and the observed spectrum g should not contain negative elements. This is imposing known a priori knowledge of $\mathbf{f}$ on the solution. The constraints on non-negative elements in the minimization problem are essential and turn out to be highly effective as a means of regularization. With non-negativity included the minimization problem (6) may be written as

$$
\min _{f \geq 0}\|\mathbf{H} \mathbf{f}-\mathbf{g}\|_{2}^{2}+\alpha\left\|\mathbf{f}^{(\mathbf{k})}\right\|_{2}^{2}
$$

The projected Newton method ${ }^{25}$ can be used to solve the above nonlinear minimization problem. The $j$ th iteration of the projected Newton algorithm can be described as follows:

$$
\begin{aligned}
& {\left[\mathbf{D}_{\mathbf{A}}+\mathbf{D}_{\mathbf{I}}\left(\mathbf{H}^{T} \mathbf{H}+\alpha \mathbf{L}^{(\mathbf{k})}\right) \mathbf{D}_{\mathbf{I}}\right] \mathbf{s}_{\mathbf{j}+1}=\mathbf{z}\left(\mathbf{f}_{\mathbf{j}}\right),} \\
& \mathbf{f}_{\mathbf{j}+1}=\left[\mathbf{f}_{\mathbf{j}}+\mathbf{s}_{\mathbf{j}+1}\right]_{+},
\end{aligned}
$$

where $\mathbf{z}\left(\mathbf{f}_{\mathbf{j}}\right)=\left(\mathbf{H}^{T} \mathbf{H}+\alpha \mathbf{L}\right) \mathbf{f}_{\mathbf{j}}-\mathbf{H}^{T} \mathbf{g},\left([\mathbf{x}]_{+}\right)_{i}=x_{i}$ if $x_{i} \geqslant 0$, and 0 otherwise, and, $\mathbf{D}_{\mathbf{A}}$ is a diagonal matrix whose $i$ th diagonal entry is 1 if the $i$ th entry of both $\mathbf{f}_{\mathbf{j}}$ and $\mathbf{z}\left(\mathbf{f}_{\mathbf{j}}^{\mathbf{k}}\right)$ are nonnegative, and is 0 otherwise. Similarly, $\mathbf{D}_{\mathbf{I}}$ is a diagonal matrix given by $\mathbf{I}-\mathbf{D}_{\mathbf{A}}$. In each of the projected Newton iterations, the linear system in Eq. (9) can be solved by the conjugate gradient method effectively. ${ }^{29}$

\section{Monte Carlo simulation of CDBS data}

There has been a significant amount of research which aims at determining the optimal regularization parameter in generalized least-square problems. ${ }^{30}$ In this work, this parameter is optimized by interactive selection, as shown schematically in Fig. 2. First, Monte Carlo (MC) CDBS spectra are generated. As outlined below, this technique closely mimics the response of a CDBS spectrometer to annihilation photons having the typical MDAP characteristic of a metal. The deconvoluted function $\mathbf{f}_{\alpha}$ is finally compared with the underlying MDAP of the material $\mathbf{f}$ through observation of the weighed residuals $\left(\mathbf{f}_{\alpha}-\mathbf{f}\right) \cdot \mathbf{w}, \mathbf{w}$ being the column vector with elements $1 / \sqrt{f_{i}}$. The "optimal" regularization parameter

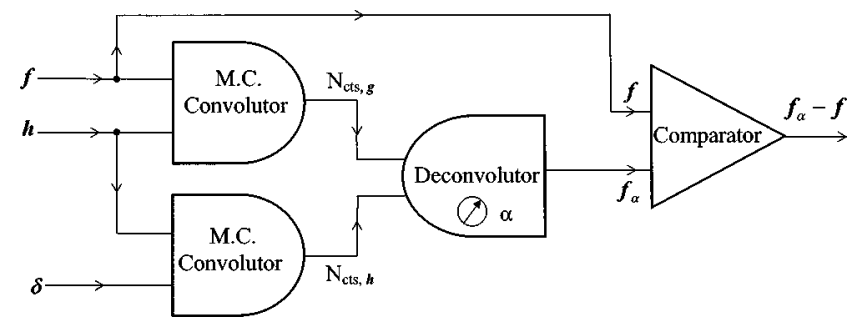

FIG. 2. Schematic diagram shows the methodology used for optimizing the deconvolution algorithm. Two runs of a Monte Carlo (MC) program simulate both the experimental processes producing a synthetic CDBS spectrum of known MDAP $\mathbf{f}$ and resolution function $\mathbf{h}$. These data are then fed into the deconvolution program (2DNNGLSD) to produce the deconvoluted $\mathbf{f}_{\alpha}$. The final weighted residual spectrum between $\mathbf{f}$ and $\mathbf{f}_{\alpha}$ is constructed for purposes of assessing the fidelity of the deconvolution. 

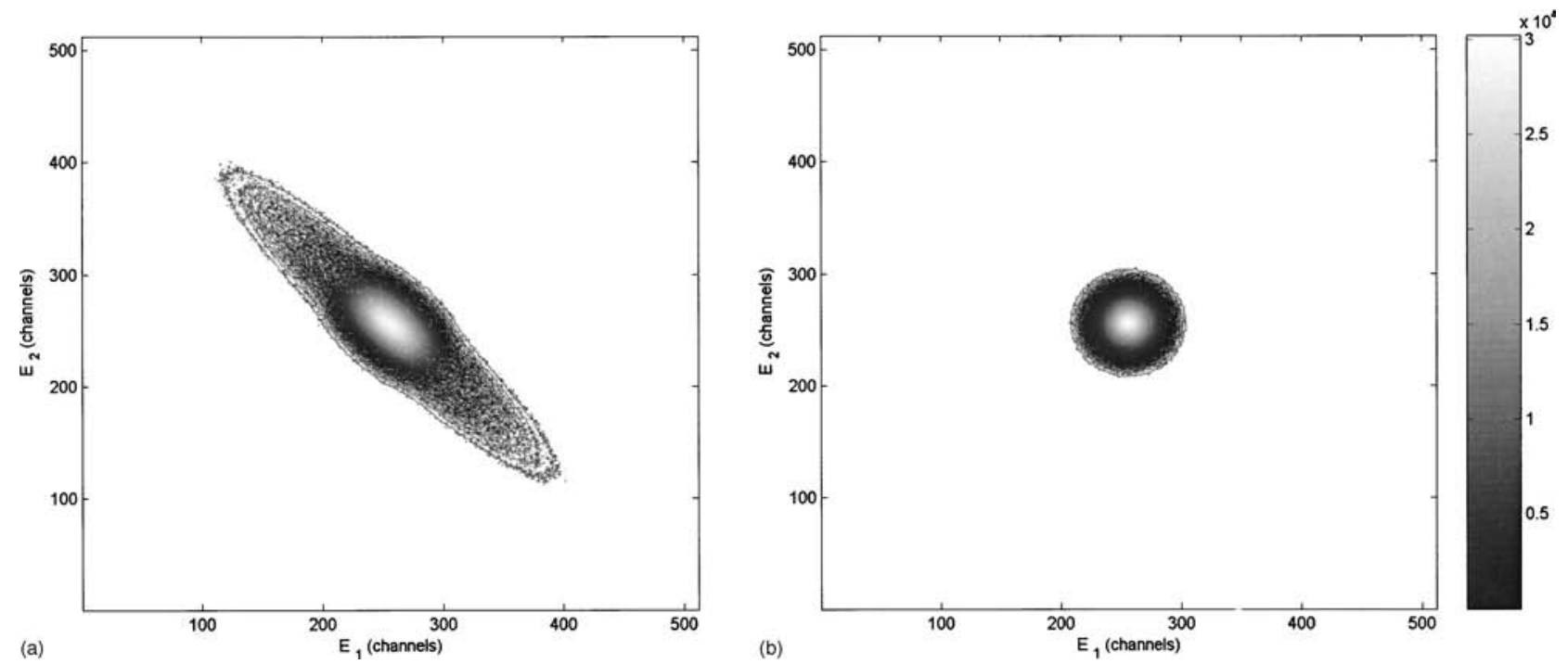

FIG. 3. Typical synthetic spectra produced by the MC simulation program. (a) The CDBS spectrum, g, is approximately corresponding to Li metal; (b) resolution function $\mathbf{h}$.

can thus be found so as to bring the closest visual match of $\mathbf{f}_{\alpha}$ to $\mathbf{f}$, or alternatively by defining a weighted "chi-squared" error:

$$
\chi^{2}=\sum_{i=1}^{N} \frac{\left(f_{\alpha, i, w}-f_{i}\right)^{2}}{f_{i}} .
$$

The method of producing the simulated data has already been described in a previous article. ${ }^{24}$ Only a brief summary will be given here. Seven variates in the range of $0-1$ are required to register a single event on the simulated $2 \mathrm{D}$ CDBS histogram. Considering the sample to be a simple metal (lithium is used here), the first variate decides whether the positron is to annihilate with a core or conduction-band electron. If the annihilation is from a core electron the next two variates are used to position its energy $E_{\gamma 1}$ according to the Gaussian distribution:

$$
P\left(E_{\gamma 1}\right)=\frac{1}{a_{C} \sqrt{2 \pi}} e^{-\left[\left(E_{\gamma 1}-E_{0}\right)^{2} / 2 a_{C}^{2}\right]},
$$

where $E_{0}=m c^{2}$ and $a_{C}$ is the standard deviation of the distribution. This distribution is obtained using the standard MC formulation for Gaussian generation: ${ }^{31,32}$

$$
E_{\gamma 1}=E_{0}+a_{C} \sqrt{2 \ln \frac{1}{\xi_{2}}} \cos 2 \pi \xi_{3},
$$

$\xi_{2}$ and $\xi_{3}$ being the two random variates. Alternatively, in the case of an event coming from the conduction band, then $E_{\gamma 1}$ is thrown according to the inverted parabola:

$$
P\left(E_{\gamma 1}\right)=\frac{3}{4 a_{F}^{3}}\left[a_{F}^{2}-\left(E_{\gamma 1}-E_{0}\right)^{2}\right],
$$

where $a_{F}$ represents the Doppler shift corresponding to the Fermi momentum. The MC method for generating Eq. (14) is not well documented, but this distribution can simply be obtained by throwing events in momentum space out to a radius of $a_{F}$ (which also closely mimics the real annihilation process in a metal). That is, one writes ${ }^{33}$

$$
E_{\gamma 1}=E_{0}+a_{F}^{3} \sqrt{\xi_{2}}\left(2 \xi_{3}-1\right),
$$

$\xi_{2}$ and $\xi_{3}$ being the two random variates. With the $E_{\gamma 1}$ energy determined, energy conservation through Eq. (1) demands that

$$
E_{\gamma 2}=2 E_{0}-E_{\gamma 1} \text {. }
$$

The next step is to incorporate the resolution broadening associated with both detectors and their associated electronics. This is implemented by shifting the energies of the annihilation event to $E_{\gamma 1}$ and $E_{\gamma 2}$ to new values according to independent randomized Gaussian distributions. Four variates are used at this stage - two for each Gaussian. The instrumental "blurring" function can be obtained easily using the same MC code by putting $a_{C}=a_{F}=0$ (i.e., by replacing h with a delta function-see Fig. 2). Finally, the events are cumulated and binned.

Various sizes of 2D matrices have been investigated $(256 \times 256,512 \times 512$, and $1024 \times 1024)$. MC spectra were thrown from $10^{4}$ to $10^{8}$ counts. To approximately mimic the ACAR momentum distribution, parameters pertinent to lithium data ${ }^{34}$ were chosen (i.e., $a_{C}=7.5 \mathrm{mrad}, a_{F}$ $=4.4 \mathrm{mrad}$ ). The $\sigma$ value of the detector was taken as $2 \mathrm{mrad}$ (corresponding to a FWHM unit of $1.2 \mathrm{keV}$ and $4.7 \mathrm{mrad}$ of ACAR equivalent). Simulated CDBS data, assuming the MDAP for Li, are shown in Fig. 3(a). The spectrum is quite ideal when compared to a real CDBS spectrum. In particular, it has no low level random background and none of the characteristic "cross" events at $E_{\gamma 1}=E_{\gamma 2}=511 \mathrm{keV}$. An example of the "blurring" function is shown in Fig. 3(b).

\section{RESULTS AND DISCUSSION}

\section{A. Improvement with non-negativity constraints}

The deconvolution of the simulated spectrum without non-negativity constraints is shown in Fig. 4. While the resulting deconvoluted image is indeed sharper, it is seen to suffer from ripples, possessing negative values, and a frag- 


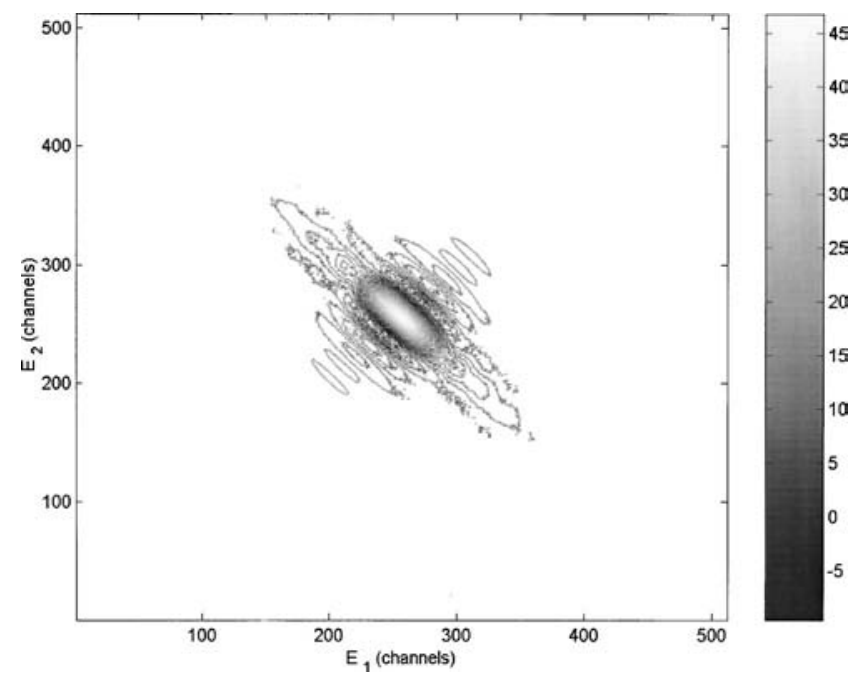

FIG. 4. Result of deconvoluted CDBS $\mathbf{f}_{\alpha}$ where no non-negativity constraint has been employed. The regularization parameter has been optimized but still negative portions exist with considerable rippling.

mented background. Variation of the regularization parameter was unable to reduce this rippling effect. It was found, however, that introduction of the non-negativity constraints into the deconvolution algorithm could largely reduce these undesirable features. The deconvoluted spectrum of a simulated CDBS data for lithium metal using non-negativity constraints with $\alpha=10^{-3}$ is shown in Fig. 5. The $\mathbf{f}_{\alpha}$ data lie close to the line $E_{1}+E_{2}=2 E_{0}$ (=512 channels) in a narrow band without any of the negative ripples present in the "single shot" method. The quality of the retrieval was assessed by taking a restricted channel cut along the diagonal line. The retrieved CDBS spectrum $\mathbf{f}_{\alpha}$ is shown in Fig. 6 together with the true MDAP function $\mathbf{f}$ and experimental 1D-ACAR data for Li metal. ${ }^{34}$ The good agreement between f and $\mathbf{f}_{\alpha}$ suggests that the non-negativity constraints are highly effective at producing accurate regularized solutions for the deconvolution. Although there is still some rippling

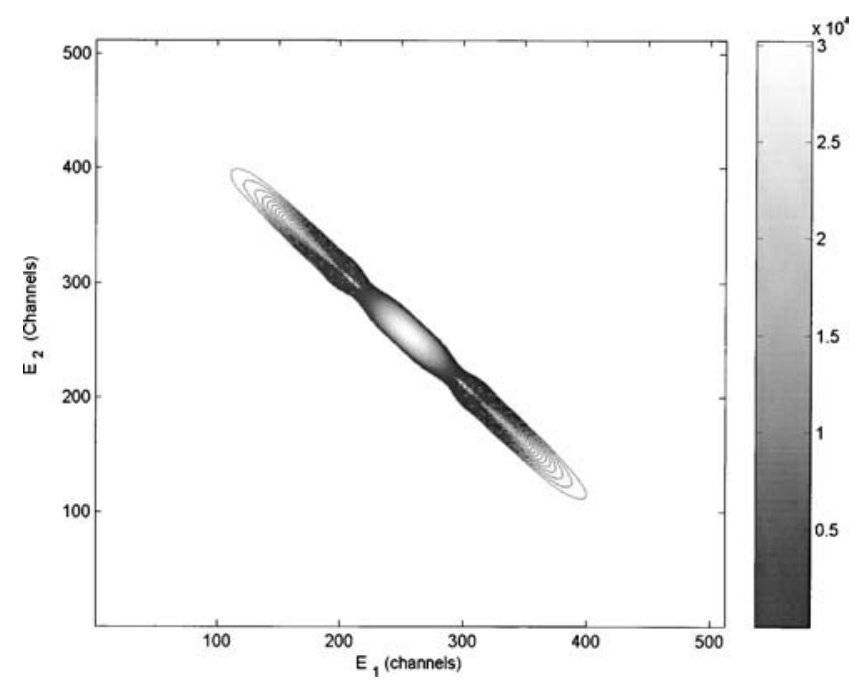

FIG. 5. Result of deconvoluted CDBS $\mathbf{f}_{\alpha}$ with non-negativity constraint has been employed. The amount of rippling in the deconvoluted result is seen to be very much reduced. For this deconvolution $\alpha=10^{-3}$ and the zeroth norm regularizer were used.
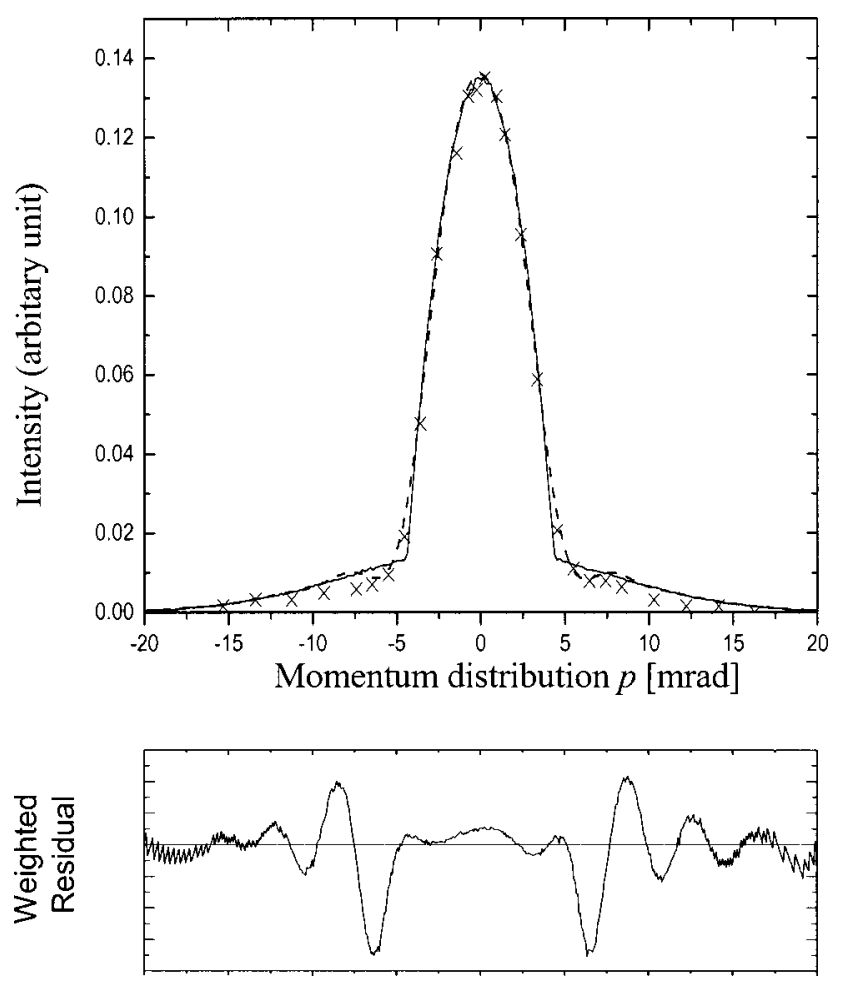

FIG. 6. 1D CDBS spectrum $\mathbf{f}_{\alpha}$ (dashed line) taken from a diagonal cut of 31 channels (two system FWHMs) compared with the true MDAP f. The crosses show ACAR-MDAP data taken from Lang et al. (Ref. 34).

present, $\mathbf{f}_{\alpha}$ does not significantly deviate from the true $\mathbf{f}$. Such reasonable agreement would be sufficient for a wide range of positron annihilation investigations.

The first problem to address in optimal deconvolution is to decide on the best cut for the channel width " $\delta$." Figure 7 shows a plot of $\chi^{2}$ against $\delta$. For low-count spectra, i.e., $N_{\text {cts }}=10^{4}$ and $10^{5}$, the minimum $\chi^{2}$ is attained when $\delta$ $=3.5$ (in FWHM units). For high-count spectra with $N_{\text {cts }}$

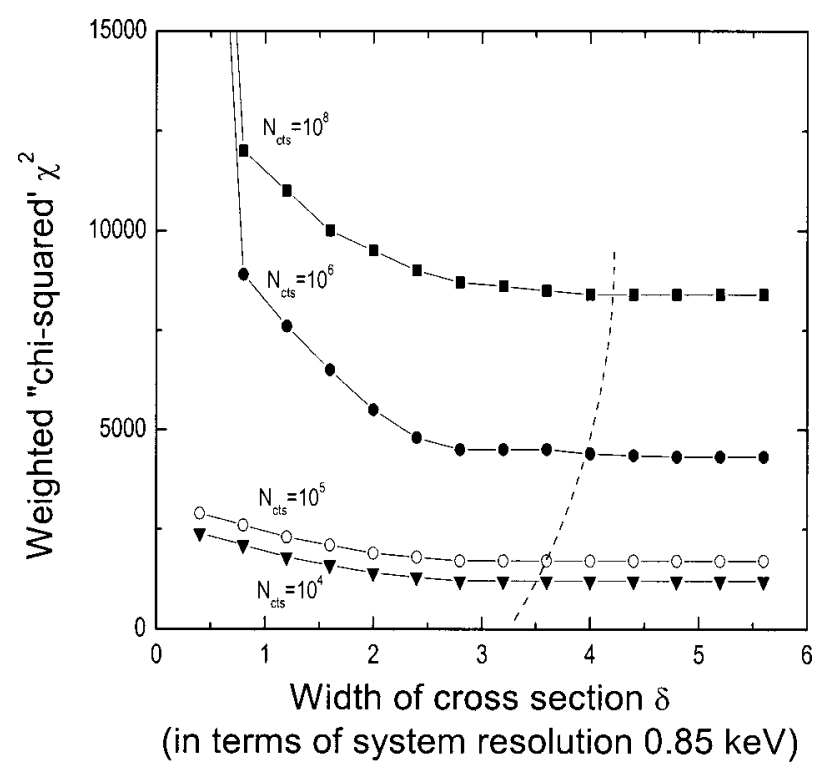

FIG. 7. Variation of summed weighted squared residuals, chi-squared, $\chi^{2}$ plotted as a function of the cutting width $\delta$ along the CDBS diagonal for spectra having $N_{\text {cts }}=10^{4}, 10^{5}, 10^{6}$, and $10^{8}$ counts. The dotted line indicates the value of $\delta$ for which $\chi^{2}$ is minimum. 


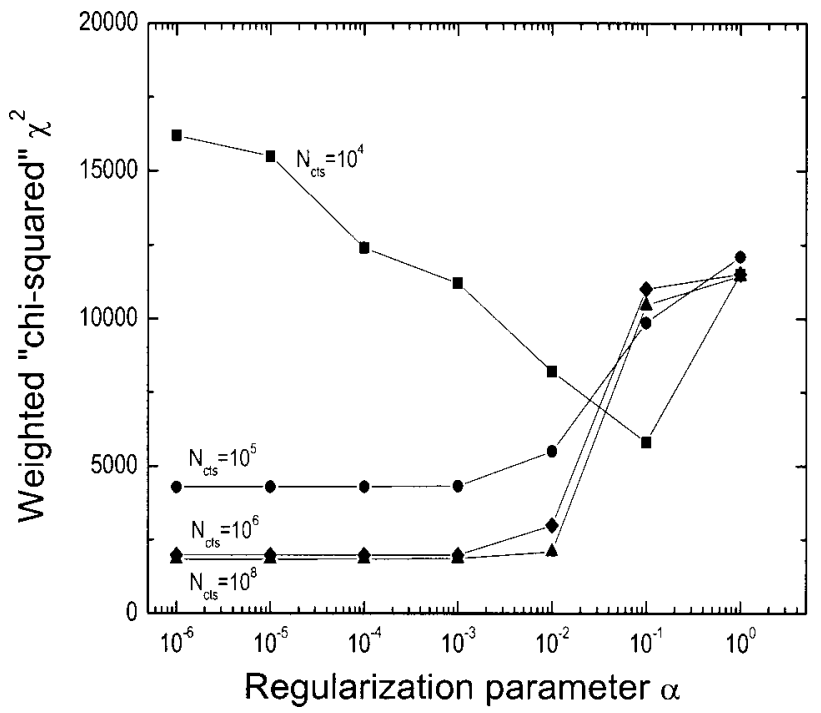

FIG. 8. Variation of the chi-squared $\chi^{2}$ of residuals plotted against the regularization parameter $\alpha$ for synthetic CDBS spectra having $=10^{4}, 10^{5}, 10^{6}$, and $10^{8}$ counts, deconvoluted using the 2DNNGLSD algorithm. The zeroth norm regularization was used, with $N_{\mathrm{chn}}=512$.

$=10^{6}$ and $10^{8}$; the $\delta$ for $\chi^{2}$ minimum is slightly larger at $\sim 4.0$ FWHM unit. These results are in good agreement with the work of Gebauer et al. ${ }^{35}$ indicating that a choice of channel width of around 2 FWHM unit is suitable for a wide range of $N_{\text {cts }}$. The value of $\delta=2$ FWHM was taken in the following optimization studies.

\section{B. Optimizing the regularization parameter and norm}

In the present study $N_{\mathrm{cts}}$ was set at either $10^{4}, 10^{5}, 10^{6}$, and $10^{8}$ while image matrix sizes $\left(N_{\text {chn }} \times N_{\text {chn }}\right)$ were set to $256 \times 256,512 \times 512$, and $1024 \times 1024$ - our computational limit. In all the studies $N_{\text {cts }}=10^{8}$ was chosen for $\mathbf{h}$ spectra and the tolerances of errors on the conjugate gradient method was taken as $10^{-3}$.

Figure 8 shows $\chi^{2}$ as a function of the regularization parameter $\alpha$. It is seen that a minimum occurs when $N_{\text {cts }}$ $=10^{4}$, while for $N_{\mathrm{cts}}=10^{5}, 10^{6}$, and $10^{8}$, the $\chi^{2}$ decreases monotonically as $\alpha$ is reduced. If $\alpha$ is too small, then insufficient regularization will cause $\chi^{2}$ to be high. Conversely, if $\alpha$ is too large, regularization errors will occur again forcing $\chi^{2}$ to be high. ${ }^{36}$ In the $N_{\text {cts }}=10^{4}$ case, the minimum occurs at $\alpha=0.1$. The absence of a minimum for the high-count spectra $\left(N_{\mathrm{cts}}=10^{5}, 10^{6}\right.$, and $\left.10^{8}\right)$ indicates the strong regularizing action of the non-negativity constraint. At $N_{\text {cts }} \geqslant 10^{5}$, the signal-to-noise ratio is so small that the effect of regularization becomes insignificant, and thus by putting $\alpha=0$, we can still obtain optimal deconvolution.

The quality of the deconvolution as expressed by $\chi^{2}$ improves as $N_{\text {cts }}$ increases since the statistical noise level reduces for large $N_{\text {cts }}$ to allow better function retrieval. Larger matrix sizes are also expected to yield better deconvolution results as more information is present in the spectra on both $\mathbf{g}$ and $\mathbf{h}$ functions. In Figs. 9(a) and 9(b) $\chi^{2}$ is plotted for different $N_{\text {chn }}$ against $\alpha$ for both $N_{\text {cts }}=10^{4}$ and $10^{8}$, respectively. Figure 9(a) shows, as already discussed, the regularization parameter $\alpha$ is still effective, the value of $\alpha=0.1$ giving optimal regularization irrespective of $N_{\mathrm{chn}}$. The important point, however, is that the $\chi^{2}$ value decreases with $N_{\text {chn }}$, indicating that some improvement in deconvolution is to be obtained by choosing higher binning levels. Irrespective of matrix sizes, the high statistics data $\left(N_{\text {cts }}=10^{8}\right)$, as shown in Fig. 9(b), produce the same "no minimum" behavior as remarked on above. Once again, the $N_{\mathrm{cts}}=10^{4}$ data give a poorer quality deconvolution. The general trend of the larger $N_{\text {chn }}$ giving smaller $\chi^{2}$ is clear.

In Figs. 10(a) and 10(b) equivalent $\chi^{2}$ vs $\alpha$ plots are shown for the cases of $k=1$ and $k=2$, respectively. A comparison of Figs. 8 and 10(a) shows very little difference between $k=0$ and $k=1$ norms. However, for the $k=2$ norm shown in Fig. 10(b), while being very similar to $k=0$ and 1 for low $\alpha\left(<10^{-2}\right)$, gives a markedly improved regularization for large regularization parameters $\alpha\left(>10^{2}\right)$. For $N_{\text {cts }}$ $=10^{5}$, there exists a $\chi^{2}$ minimum now at $\alpha=0.1$, and a better deconvolute is obtained than the case of zero $\alpha$. From this evidence it is to be generally concluded that the second derivative is a slightly better choice of norm. ${ }^{23}$ However, for high spectral content CDBS data $N>10^{5}$, in which the regularization parameter $\alpha \leqslant 10^{-4}$, there is no noticeable difference in the quality of deconvolution, all derivatives essentially yielding the same result. This finding is the same as that of Chambless and Broadway, ${ }^{21,22}$ who also used nonnegativity constraints in deconvolution.

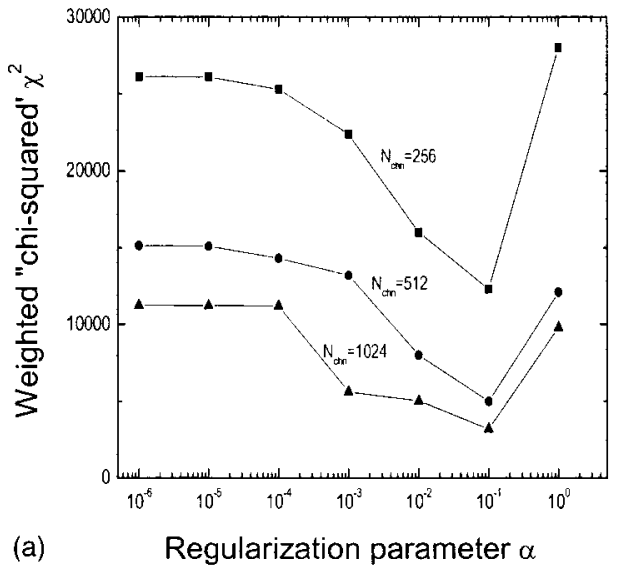

(a)

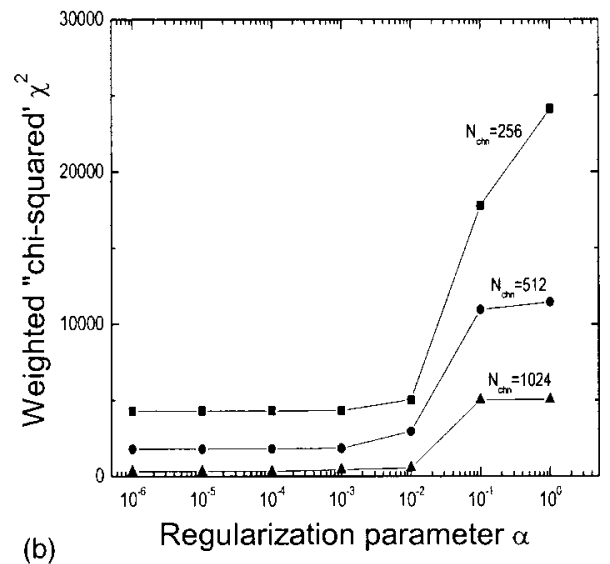

(b)
FIG. 9. Variation of the chi-squared $\chi^{2}$ of residuals plotted against the regularization parameter $\alpha$ for synthetic CDBS spectra obtained using the 2DNNGLSD algorithm; (a) with $N_{\text {cts }}=10^{4}$ and (b) with $N_{\text {cts }}=10^{8}$. The data are shown for the different CDBS matrix sizes or $N_{\text {chn }}=256,512$, and 1024. 


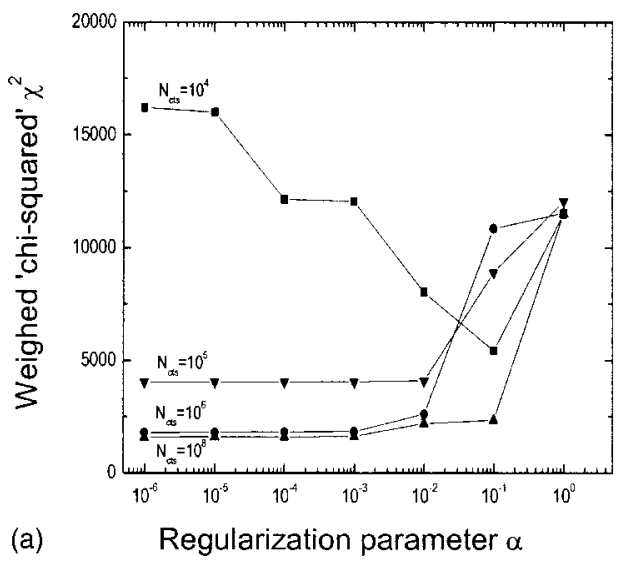

\section{Residual instrumental function (RIF)}

With many modern spectroscopy systems the distinction between hardware and software components becomes less distinct, since both contribute to the final data quality. Certainly within the context of the present discussion of deconvolution, a CDBS spectroscopy system may most appropriately be considered to consist of the hardware electronics and software as a single system. This being the case, the single system may be expected to have an effective resolution function, termed the residual instrumental function (RIF), ${ }^{19}$ which is sharper than that of the hardware alone, namely, the hardware instrumental function (HIF). In order to address this question quantitatively, a deconvolution improvement factor $F$ is defined as

$$
F=\frac{\text { FWHM }(\text { HIF })}{\text { FWHM }(\text { RIF })} .
$$

Comparison of the narrower width of deconvoluted CDBS data from the peak along the $E_{1}-E_{2}=0$ diagonal in Fig. 4 and the raw data in Fig. 1(a) reveals an improvement of the effective system resolution. This follows from Eq. (1) since the detection errors $\delta_{1}$ and $\delta_{2}$ add in quadrature along this diagonal, making the FWHM in this direction an accurate indicator of the system resolution. Some caution must be taken, however, in generally assessing the system resolution from the FWHM value along the $E_{1}-E_{2}=0$ (positive) diagonal since the shape of the combined resolution curve along this diagonal is not, strictly speaking, the same as that along the $E_{1}-E_{2}=2 m c^{2}$ (negative) diagonal. As discussed by Britton et al. ${ }^{7}$ the resolution function along the negative diagonal (which is the resolution function of importance in CDBS) is the correlation integral of the two separate detector resolution functions $R_{1}(E)$ and $R_{2}(E)$, while that along the positive diagonal is the convolution of $R_{1}(E)$ and $R_{2}(E)$. In the case of $R_{1}(E)$ and $R_{2}(E)$ being both symmetric functions, as is the case in the present testing on simulated spectra, the combined resolution function is the same along both diagonals. Thus, in the present study we are permitted to take FWHM values of HIF and RIF from the profiles of the raw and deconvoluted data along the positive diagonal as a means of assessing the quality of the deconvolution.

The $E_{1}-E_{2}=0$ diagonal cross sections of both $\mathbf{g}$ and $\mathbf{f}_{\alpha}$ data are shown in the inset of Fig. 11. The value of $F$ obtained from these cross sections is plotted against the regu- larization parameter $\alpha$ for the simulated $512 \times 512$ channel Li CDBS spectra of different $N_{\text {cts }}$ in Fig. 11. For $\alpha<10^{-3}, F$ remains essentially constant. (The lowering of $F$ for large $\alpha$ results from the regularization error tends to broaden $\mathbf{f}_{\alpha}$ through oversmoothing.) Apart from the $N_{\text {cts }}=10^{3}$ case, the saturation value of $F$ is $\sim 3$. An increase $N_{\text {cts }}$ improves $F$ as expected. However, the reason for the small improvement (i.e., about 3.0-3.1 for $N_{\text {chn }}=512$ ) and the small increase in RIF quality against a large increases of $N_{\text {cts }}$ is not well understood. In a similar study, Beling et al. ${ }^{8}$ using Monte Carlo data with a much simpler Stoke's scheme found empirically that

$$
F=1.5+0.067 \ln \left(N_{\text {cts }} N_{\text {chn }}\right) .
$$

This relationship gives a stronger dependency of $F$ on $\ln \left(N_{\mathrm{cts}}\right)$ than that found in the present work, although the magnitude, i.e., $F \sim 3$ is similar. It is easy to obtain $F$ values of around 3 from deconvoluted spectrum, and this leads to some optimism for approaching typical ACAR resolution performance using CDBS. In our case, the effect of depen-

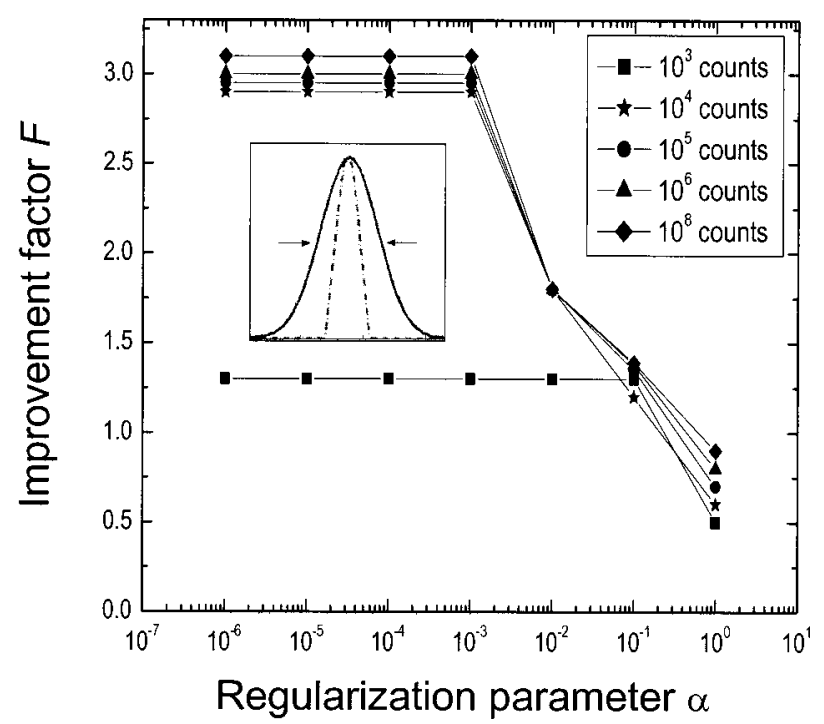

FIG. 11. Deconvolution improvement factor $F$ plotted as a function of regularization parameter $\alpha$ for zeroth norm regularization obtained using the 2DNNGLSD algorithm. The data are shown for $N_{\mathrm{cts}}=10^{3}, 10^{4}, 10^{5}, 10^{6}$, and $10^{8}$ counts. The inset shows the cross sections through the CDBS diagonal for both the $\mathbf{g}$ and $\mathbf{f}_{\alpha}$ functions (the ratio's of which the FWHMs define $F$ ). 

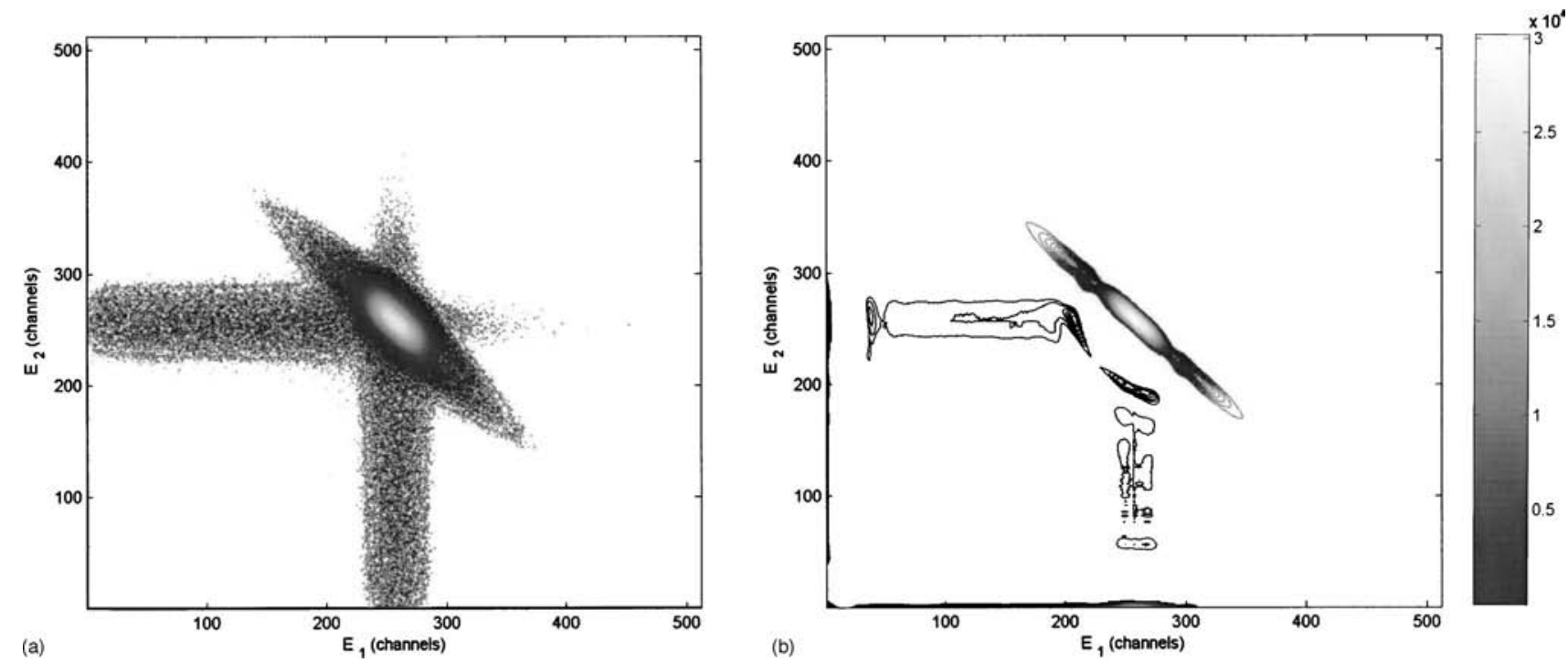

FIG. 12. (a) Experimental CDBS spectrum for polycrystalline Al metal, with high energy backgrounds subtracted across the whole spectrum. (b) The same data after having been deconvoluted using the 2DNNGLSD algorithm. The $\mathbf{h}$ function (not shown) was that of Fig. 1(b), but again the cross pieces were partially subtracted based upon the data on the high energy sides. The zeroth norm with $\alpha=10^{-3}$ was used in this deconvolution. The number of spectral counts $N_{\mathrm{cts}}=2 \times 10^{7} \mathrm{cts}$.

dence on $N_{\text {cts }}$ and $N_{\text {chn }}$ on the quality of deconvolution is less significant, and the realistic gains in instrumental resolution to be obtained using the non-negativity constraint by increasing $N_{\text {cts }}$ and $N_{\text {chn }}$ are more limited.

Deconvoluted spectra of effective system resolution (FWHM) $320 \mathrm{eV}$ (or $1.25 \mathrm{mrad}$ ACAR equivalent) are obtained with $F \sim 3$ from the present simulated data, which started with a $\mathrm{HP}(\mathrm{Ge})$ detector resolution of (FWHM) 1200 $\mathrm{eV}$. The comparison with the resolving power of a typical ACAR spectrometer $\sim 0.5-1.0 \mathrm{mrad}$ (i.e., $128-256 \mathrm{eV}$ ) is encouraging. It must also be stressed that while the nonnegativity method is good, it is probably not the ultimate optimal way of performing CDBS spectral deconvolutions. Further improvements may well be obtained with more sophisticated algorithms currently under development in our research group.

\section{Deconvoluting experimental CDBS spectra}

The CDBS spectrometer used in this work consisted of two HPGe detectors, with $80 \%$ and $60 \%$ relative efficiencies and 1.2 and $2.0 \mathrm{keV}$ FWHM resolutions at $514 \mathrm{keV}$. The net effective resolution of the system was $\sim 4.0 \mathrm{mrad}$. The number of counts in the CDBS spectrum $\mathbf{g}$ and the resolution function spectrum $\mathbf{h}$ (obtained from ${ }^{85} \mathrm{Sr}$ in quasicoincidence) were $2 \times 10^{7}$ and $5 \times 10^{7}$, respectively. Digital spectrum stabilization was employed on both $E_{1}$ and $E_{2}$ channels.

The experimental CDBS data for annealed polycrystalline aluminum are shown in Fig. 12(a), where background counts due to Compton events from the $1.27 \mathrm{MeV}$ gamma ray associated with ${ }^{22} \mathrm{Na}$ have been subtracted off by fitting a Gaussian to the "cross" data on the high energy sides of both $E_{1}$ and $E_{2}$. (This background was not considered to be part of the experimental signal in contrast to the "tailing" counts on the low energy side of the "cross," which may be considered as part of the instrumental resolution. The back- ground was similarly subtracted from the $\mathbf{h}$ spectrum). The deconvoluted $\mathbf{f}_{\alpha}$ is shown in Fig. 12(b). The improved resolution is clearly seen from the narrowed width of the data in the $E_{1}-E_{2}=0$ direction. Moreover, there is no sign of rippling in the near vicinity of this CDBS diagonal. Unfortunately, there is some residual signal belonging to the low
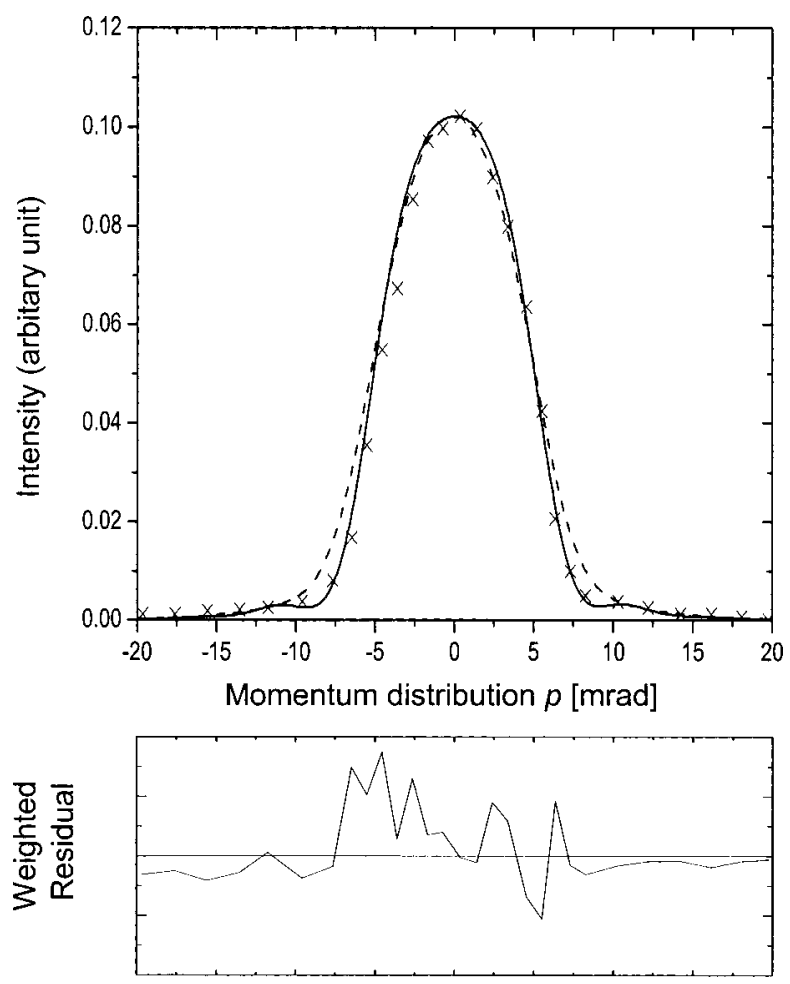

FIG. 13. 1D CDBS cut (with channel width=2 system FWHMs) for the deconvoluted Al data shown in Fig. 12(b). The dotted line shows the CDBS cut prior to deconvolution. The solid line shows the deconvoluted $\mathbf{f}_{\alpha}$. The crosses show the 1D-ACAR data of Lang et al. (Ref. 34) for polycrystalline Al. The residual plot shows an accurate MDAP produced over the whole momentum range after deconvolution. 
energy "cross" portions of $E_{1}$ and $E_{2}$. This effect indicates that the low energy "cross" portions in the function $\mathbf{h}$ give a perfect description of the instrumental function. The reasons for this are unclear at present. This residual, however, is unlikely to be affecting the data on the main CDBS diagonal, which is seen to be nicely symmetric.

The quality of the deconvolute can be assessed by comparing it with the known $2 \gamma$ momentum density as obtained using 1D-ACAR data for Al. This comparison is shown in Fig. 13. The ACAR data were taken from the early work of Lang et ll. $^{34}$ and are used here for comparison, being the only data available for the polycrystalline metal. The agreement with the ACAR data, while not perfect, is seen to be very good. Indeed, we believe that for many research applications this quality of $2 \gamma$ momentum density may prove quite adequate.

\section{ACKNOWLEDGMENTS}

The work described in this article is partially supported by grants from the Research Grant Council (RGC) of the Hong Kong Special Administrative Region, China (under Project Nos. HKU7103/02P, HKU7104/98P, HKU7091/00P, and HKU1/00C) and the Hung Hing Ying Physical Science Research Fund. The research of one of the authors (M.K.N.) is supported in part by RGC Grant Nos. 7130/02P and 7046/ 03P and a Science Faculty Collaborative Seed Grant, 20022003. The authors wish to thank C. M. Woo for writing the MPI code on the high performance computer cluster and P. Y. Kwan for preparing the graphs.

${ }^{1}$ M. Sob, H. Sormann, and J. Kuriplach, Adv. Quantum Chem. 42, 77 (2003).

${ }^{2}$ R. N. West, in Positron Spectroscopy of Solids, edited by A. Dupasquier and A. P. Mills, Jr. (IOS, Ohmsha, 1995), p. 75.

${ }^{3}$ L. Liszkay, C. Corbel, L. Baroux, P. Hautojarvi, M. Bayhan, A. W. Brinkman, and S. Tararenko, Appl. Phys. Lett. 64, 1380 (1994).

${ }^{4}$ M. Alatalo, H. Kauppinen, K. Saarinen, M. J. Puska, J. Makinen, P. Hautojarvi, and R. M. Nieminen, Phys. Rev. B 51, 4176 (1995).

${ }^{5}$ P. Asoka-Kumar, M. Alatalo, V. J. Ghosh, A. C. Kruseman, B. Nielsen, and K. G. Lynn, Phys. Rev. Lett. 77, 2097 (1996).

${ }^{6}$ J. R. MacDonald, K. G. Lynn, R. A. Boie, and M. F. Robbins, Nucl. Instrum. Methods 153, 189 (1977).

${ }^{7}$ D. T. Britton, W. Junker, and P. Sperr, Mater. Sci. Forum 105, 1845 (1992).
${ }^{8}$ C. D. Beling, M. Li, Y. Y. Shan, S. H. Cheung, S. Fung, B. K. Panda, and A. P. Seitsonen, J. Phys.: Condens. Matter 10, 10475 (1998).

${ }^{9}$ A. R. Stokes, Proc. Phys. Soc. London 61, 382 (1948).

${ }^{10}$ R. Chang R., B. Williams, and M. Copper, Philos. Mag. 23, 115 (1971).

${ }^{11}$ J. P. Schaffer, E. J. Shaughnessy, and P. L. Jones, Nucl. Instrum. Methods Phys. Res. B 5, 75 (1984).

${ }^{12}$ J. P. Schaffer and P. L. Jones, J. Phys. F: Met. Phys. 16, 1885 (1986).

${ }^{13}$ Y. Kong and K. G. Lynn, Nucl. Instrum. Methods Phys. Res. A 302, 145 (1991).

${ }^{14}$ A. Schukla, M. Peter, and L. Hoffmann, Nucl. Instrum. Methods Phys. Res. A 335, 310 (1993).

${ }^{15}$ Shizuma Kiyoshi, Nucl. Instrum. Methods 150, 447 (1978).

${ }^{16}$ Y. Ichioka, Y. Takubo, K. Matsuoka, and T. Suzuki, J. Opt. (Paris) 12, 35 (1981).

${ }^{17}$ S. Dannefaer and D. P. Kerr, Nucl. Instrum. Methods 131, 119 (1975).

${ }^{18}$ L. Calderin and J. J. Diaz, Nucl. Instrum. Methods Phys. Res. B 117, 457 (1996).

${ }^{19}$ A. A. Manuel, in Proceedings of the International School of Physics, Enrico Fermi, Societa Italiana di Fisica (IOS, Bologna, Italy, 1995), p. 155.

${ }^{20}$ P. Paatero, S. Manninen, and T. Paakkari, Report No. 75, University of Helsinki (1974).

${ }^{21}$ D. A. Chambless and J. A. Broadway, IEEE Trans. Nucl. Sci. 2, 1938 (1981).

${ }^{22}$ D. A. Chambless and J. A. Broadway, Nucl. Instrum. Methods 179, 563 (1981).

${ }^{23}$ B. K. Panda, S. Fleischer, C. C. Ling, C. D. Beling, S. Fung, and S. Panda, Appl. Surf. Sci. 85, 182 (1997).

${ }^{24}$ K. F. Ho, K. P. Ng, C. D. Beling, S. Fung, K. L. Chan, and H. W. Tang, Mater. Sci. Forum 363, 673 (2001).

${ }^{25}$ D. P. Bertsekas, Constrained Optimization and Lagrange Multiplier Methods (Academic, New York, 1982), pp. 76 and 221-246.

${ }^{26}$ J. H. Jansson, Deconvolution of Images and Spectra (Academic, New York, 1984).

${ }^{27}$ A. Tikhonov and V. Arsenine, Methodes de Resolution de Problemes Mal Poses (Mir, Moscow, 1976).

${ }^{28}$ M. K. Ng, R. H. Chan, and W. C. Tang, SIAM J. Sci. Comput. (USA) 21, 851 (1999).

${ }^{29}$ C. R. Vogel, Scientific Computing (Springer, Singapore, 1997), p. 148.

${ }^{30}$ G. Wahba, SIAM (Soc. Ind. Appl. Math.) J. Numer. Anal. 14, 651 (1977).

${ }^{31}$ L. L. Carter and E. D. Cashwell, Particle Transport Simulation with the Monte Carlo Method (USERDA Technical Information Center, Oak Ridge, TN, 1975).

${ }^{32}$ G. S. Fisherman, Principles of Discrete Event Simulation (WileyInterscience, New York, 1978).

${ }^{33}$ P. K. MacKeown and C. D. Beling (private communication).

${ }^{34}$ G. Lang, S. de Benedetti, and R. Smoluchowski, Phys. Rev. 99, 596 (1955).

${ }^{35}$ J. Gebauer, R. Krause-Rehberg, S. Eichler, and F. Borner, Appl. Surf. Sci. 149, 110 (1997).

${ }^{36}$ R. L. Lagendijk and J. Biemond, Iterative Identification and Restoration of Images (Kluwer Academic, Boston, 1991). 